Pacific Journal of Mathematics

SOME METRICS ON THE SUBSPACES OF A BANACH SPACE 


\section{SOME METRICS ON THE SUBSPACES OF A BANACH SPACE}

\section{EARL BERKSON}

Notation, Terminology and Conventions. We shall denote by $[X]$ the Banach algebra of all bounded operators mapping a Banach space $X$ into itself. Unless otherwise stated, all operators are defined everywhere and bounded, and all convergence of operators is with respect to the uniform operator topology. An invertible element of $[X]$ will be called an invertible operator or an automorphism. A linear homeomorphism of one Banach space onto another will be called an isomorphism. The symbol " $I$ " will be used for the identity operator. The term "subspace" will mean "closed linear manifold," and given a subspace $Y$, we will denote by $\Sigma(Y)$ the set, $\{y \in Y \mid\|y\|=1\}$. Finally, the set of subspaces of a Banach space $X$ will be denoted by $S_{X}$. Additional terminology and notation will be developed as needed.

Introduction. This paper is devoted to the study of three metrics on the set of subspaces of a Banach space : one due to J. J. Schäffer (see $\$ 1$ ), one obtained as a modification of the opening (see $\S \S 2$ and 3 ), and one due to J. D. Newburgh (see §7). I am indebted to Dr. J. J. Schäffer for helpful conversations and suggestions, and for the elegance of the demonstration in $\$ 5$. In addition, it was a desire on my part to find geometric properties of his metric which led me to compare it with the opening. It turns out that this metric has strong connections with the opening. The connections between the three metrics, as well as properties of the opening of interest in themselves, form the subject matter of this paper.

1. The metric of Schäffer. In this section we list some of Schäffer's results surrounding the definition of his metric. This metric is introduced and discussed in [11].

Definition. Let $X$ be a Banach space. For arbitrary subspaces $Y, Z$ we define :

$$
r_{0}(Y, Z)=\left\{\begin{array}{l}
\text { inf }\{\|C-I\| \mid C \text { is an invertible operator and } C Y=Z\}, \\
\text { if such an operator } C \text { exists. } \\
1, \text { if no such operator } C \text { exists. }
\end{array}\right.
$$

Received March 20, 1961, and in revised form August 24, 1962. This paper is taken (in revised form) from part of the author's 1960 doctoral dissertation at the University of Chicago, where he was a National Science Foundation fellow, 


$$
\begin{aligned}
& r(Y, Z)=\max \left\{r_{0}(Y, Z), r_{0}(Z, Y)\right\} \\
& d(Y, Z)=\log (1+r(Y, Z)) .
\end{aligned}
$$

$d$ is a metric on $S_{X}$.

$$
r_{0}(Y, Z) \leqq 1 \text {. }
$$

$$
r_{0}(Y, Z)<1 \text { implies } r_{0}(Z, Y) \leqq \frac{r_{0}(Y, Z)}{1-r_{0}(Y, Z)} \text {. }
$$

$$
r_{0}(Y, Z) \leqq r(Y, Z) \leqq \frac{d(Y, Z)}{\log 2} \leqq \frac{r(Y, Z)}{\log 2} .
$$

If, in addition, $r_{0}(Y, Z)<1$, then

$$
r(Y, Z) \leqq \frac{r_{0}(Y, Z)}{1-r_{0}(Y, Z)} .
$$

(1.5) If there exist projections $P_{Y}$ and $P_{Z}$ with ranges $Y$ and $Z$, respectively, such that $\left\|P_{Y}-P_{Z}\right\|<1$, then $I-P_{Y}+P_{Z}$ is an invertible operator mapping $Y$ onto $Z$, and consequently

$$
r_{0}(Y, Z) \leqq\left\|P_{Y}-P_{Z}\right\|
$$

Definition. We shall say that a subspace is complemented provided $X$ is the direct sum of it and another subspace.

(1.6) The set of complemented subspaces is an open set relative to $d$.

2. The opening. This section is devoted to the concept of opening, which was first introduced in Hilbert space by M. G. Krein and M. A. Krasnoselski [8]. The definition given by these authors is as follows :

Let $Y$ and $Z$ be subspaces of a Hilbert space $X$. The opening of $Y$ and $Z$, which will be designated as $\theta(Y, Z)$, is defined as $\left\|P_{Y}-P_{Z}\right\|$, where $P_{Y}$ (resp., $P_{Z}$ ) is the projection on $Y$ along $Y^{\perp}$ (resp., on $Z$ along $Z^{\perp}$ ).

This definition was later extended to arbitrary Banach spaces in a paper by M. G. Krein, M. A. Krasnoselski, and D. P. Milman [9] as follows :

Let $Y$ and $Z$ be subspaces of a Banach space $X$. The opening of $Y$ and $Z, \theta(Y, Z)$, is defined by

$$
\left.\theta(Y, Z)=\max \quad \operatorname{sip}_{\substack{y \in Y \\\|y\|=1}} \rho(y, Z), \sup _{\substack{z \in Z \\\|z\|=1}} \rho(z, Y)\right\},
$$

where $\rho(x, S)$ denotes the distance of the point $x$ from the set $S$ i.e., $\inf _{s \in S}\|x-s\|$.

A proof that this definition actually extends the one originally given 
in Hilbert space may also be found in $\S 34$ of [1]. This definition of $\theta$ for an arbitrary Banach space, strictly speaking, does not make sense if one or both of $Y, Z$ is $\{0\}$. If one and only one of $Y, Z$ is $\{0\}$, we set $\theta(Y, Z)$ equal to 1 , and we also take $\theta(\{0\},\{0\})$ to be 0 .

It is evident that $0 \leqq \theta(Y, Z) \leqq 1$, and that $\theta(Y, Z)=\theta(Z, Y)$. Also, by the lemma of $F$. Riesz (see Theorem 3.12-E of [13]), if $Y$ is a proper subspace of $Z$, then $\theta(Y, Z)=1$. Roughly speaking, there are various properties which are shared by subspaces whose opening is sufficiently small. We give some sample theorems illustrating this point. Their proofs may be found in [3].

2.1. Theorem. Let $Y$ and $Z$ be subspaces of a Banach space, with $\theta(Y, Z)<1$. If one of $Y, Z$ is finite dimensional, then both have the same dimension.

2.2. Theorem. (See also $§ 34$ of [1].) If $Y$ and $Z$ are subspaces of a Hilbert space, and if $\theta(Y, Z)<1$, then $Y$ and $Z$ have the same orthogonal dimension.

2.3. THEOREM. If $Y$ and $Z$ are subspaces of a Banach space whose opening is less than $1 / 2$, then the minimum cardinality of a dense subset of $Y$ is the same as that of $Z$.

3. Uniform structure determined by $\theta$. It is evident that $\theta$ is a metric on the subspaces of a Hilbert space. However, it is not true for an arbitrary Banach space $X$ that $\theta$ is a metric on $S_{X}$. In fact, consider the real Banach space $1^{1}(2)$. For $0<a<1 / 2$, let $A$ be the subspace generated by $(1 /(a+1), a /(a+1)$. Also let $U$ and $V$ be the subspaces generated by $(1,0)$ and $(2 / 3,1 / 3)$, respectively. Then it is straightfoward to verify that $\theta(U, V)=1 / 2, \theta(U, A)=a$, and $\theta(A, V)=(1-2 a) / 2(a+1)$. Hence $\theta(U, V)>\theta(U, A)+\theta(A, V)$, and so the triangle inequality does not hold. Nonetheless $\theta$ is always quite close to being a metric; in just what sense we shall make precise presently.

The metric $\tilde{\theta}$ is defined in [4] as follows:

Definition. For subspaces $Y, Z$ of a Banach space $X$ we define $\tilde{\theta}(Y, Z)$ by

$$
\left.\tilde{\theta}(Y, Z)=\max \sup _{y \in \Sigma(Y)} \rho(y, \Sigma(Z)), \sup _{z \in \Sigma(Z)} \rho(z, \Sigma(Y))\right\} .
$$

In case one of $Y, Z$ is $\{0\}$, we complete the definition by taking $\tilde{\theta}(Y, Z)=\theta(Y, Z)$, and we point out that in the definition above $\tilde{\theta}(Y, Z)$ is the Hausdorff distance [6] between $\Sigma(Y)$ and $\Sigma(Z)$. The 
following theorem occurs in [4].

3.1. TheOREM. For $Y, Z \in S_{X}, \tilde{\theta}(Y, Z) / 2 \leqq \theta(Y, Z) \leqq \tilde{\theta}(Y, Z)$. Thus $\theta$ determines the same uniformity on $S_{X}$ as the metric $\tilde{\theta}$ (the uniformity determined by $\theta$ has as a base the family of subsets of $S_{X} \times S_{X}$ of the form $\{(Y, Z) / \theta(Y, Z)<r\}$, where $\left.r>0\right)$. We shall use the expression, "with respect to $\theta$ " instead of such expressions as "with respect to the uniformity determined by $\theta$ " and "with respect to the uniform topology induced by the uniformity determined by $\theta$ ". For our purposes, the expressions, "which respect to $\theta$ " and "with respect to $\tilde{\theta} "$, are interchangeable. In view of (1.4.) we can deal similarly with $r_{0}$ and $d$, and we shall do so.

The next theorem shows that the uniformity for $S_{X}$ determined by $\theta$ is contained in the uniformity determined by $d$, and so if a sequence of subspaces in Cauchy (resp., convergent) with respect to $d$, then it is also Cauchy (resp., convergent to the same limit) with respect to $\theta$.

3.2. THEOREM. If $X$ is a Banach space, and $Y$ and $Z$ are subspaces, then $\theta(Y, Z) \leqq r(Y, Z)$.

Proof. The nontrivial case is that in which $r(Y, Z)<1$ and neither $Y$ nor $Z$ is $\{0\}$. In this case, if $C$ is an automorphism mapping $Y$ onto $Z$, and $y \in \Sigma(Y)$, we have

$$
\rho(y, Z) \leqq\|y-C y\| \leqq\|I-C\| .
$$

Hence $\sup _{y \in \Sigma(Y)} \rho(y, Z) \leqq r_{0}(Y, Z)$, and similarly $\sup _{z \in \Sigma(Z)} \rho(z, Y) \leqq r_{0}(Z, Y)$.

If $X$ is a Hilbert space, then $\theta$ and $d$ determine the same uniformity on $S_{X}$. This observation has been made on page 563 in [11], but, in fact, we can state:

3.3. CoRollary. If $X$ is a Hilbert space and $Y$ and $Z$ are subspaces, then $r(Y, Z)=\theta(Y, Z)$.

Proof. Let $P_{Y}$ (resp., $P_{Z}$ ) be the projection on $Y$ along $Y^{\perp}$ (resp., on $Z$ along $\left.Z^{\perp}\right) . \quad \theta(Y, Z)=\left\|P_{Y}-P_{Z}\right\|$. By (1.5.), $r(Y, Z) \leqq \theta(Y, Z)$.

3.4. Corollary. If $X$ is finite dimensional, then $r_{0}$ and $\theta$ determine the same uniformity on $S_{X}$.

Proof. $X$ can be renormed with an equivalent norm which makes it a Hilbert space, 
4. Completeness of $d$ and $\tilde{\theta}$.

4.1. Theorem. Let $X$ be a Banach space. $\left(S_{X}, d\right)$ is complete.

Proof. Let $\left\{Y_{n}\right\}$ be a sequence of subspaces such that

$$
r_{0}\left(Y_{n}, Y_{m}\right) \longrightarrow 0 \text {. }
$$

We can assume without loss of generality that $r_{0}\left(Y_{n}, Y_{n+1}\right) \leqq 1 / 2^{n+1}$. For each $n$ let $C_{n}$ be an invertible operator such that:

$$
C_{n} Y_{n}=Y_{n+1}, \text { and }\left\|I-C_{n}\right\|<\frac{1}{2^{n}} .
$$

Since $\left|\left\|C_{n}\right\|-1\right| \leqq\left\|I-C_{n}\right\|<1 / 2^{n}$, for each $n$, it is clear that

$$
\sum_{n=1}^{\infty}||\left|C_{n} \|-1\right|
$$

is convergent; hence the infinite product

$$
\prod_{n=1}^{\infty}\left\|C_{n}\right\|=\prod_{n=1}^{\infty}\left\{1+\left(\left\|C_{n}\right\|-1\right)\right\}
$$

is absolutely convergent. Let $M$ be a bound for the sequence

$$
\left\{\prod_{n=1}^{k}\left\|C_{n}\right\|\right\}_{k} \text {. }
$$

We now use the following notation:

$$
\begin{aligned}
& \prod_{i=1}^{n} C_{j}=C_{n} C_{n-1} \cdots C_{2} C_{1} . \\
& \left\|\left(\prod_{j=1}^{n+1} C_{j}-\prod_{j=1}^{n} C_{j}\right)\right\|=\left\|\left(C_{n+1}-I\right)\left(\prod_{j=1}^{n} C_{j}\right)\right\| \\
& \leqq\left\|C_{n+1}-I\right\| \prod_{j=1}^{n}\left\|C_{j}\right\| .
\end{aligned}
$$

Let $T_{n}=\prod_{j=1}^{n} C_{j}$. By (4.2.) $\left\|T_{n+1}-T_{n}\right\| \leqq M / 2^{n+1}$. Thus the series $\sum_{n=1}^{\infty}\left(T_{n+1}-T_{n}\right)$ is absolutely convergent, and so $\left\{T_{n}\right\}$ converges. Let $T_{0}=\lim _{n} T_{n}$.

For each $j$,

$$
\left\|C_{j}^{-1}\right\| \leqq \frac{1}{1-\left\|I-C_{j}\right\|} \leqq \frac{1}{1-\frac{1}{2^{j}}}
$$

Consequently, for each $n$ 


$$
\left\|T_{n}^{-1}\right\|=\left\|C_{1}^{-1} C_{2}^{-1} \cdots C_{n-1}^{-1} C_{n}^{-1}\right\| \leqq \frac{1}{\prod_{\jmath=1}^{n}\left(1-\frac{1}{2^{j}}\right)} .
$$

Since $\prod_{j=1}^{\infty}\left(1-\left(1 / 2^{j}\right)\right)$ converges absolutely, we have by (4.3) :

$$
\varlimsup_{n}\left\|T_{n}^{-1}\right\|<\infty \text {. }
$$

It now follows (see Theorem 4.11.1 of [7]) that $T_{0}$ is invertible.

Let $Y_{0}=T_{0}\left(Y_{1}\right)$. Clearly for each $n, T_{n} Y_{1}=Y_{n+1}$, and so

$$
T_{0} T_{n}^{-1} Y_{n+1}=T_{0} Y_{1}=Y_{0} \text {. }
$$

Hence $T_{0} T_{n}^{-1}$ is an invertible operator mapping $Y_{n+1}$ onto $Y_{0}$. For each $n$,

$$
r_{0}\left(Y_{n+1}, Y_{0}\right) \leqq\left\|I-T_{0} T_{n}^{-1}\right\|=\left\|\left(T_{n}-T_{0}\right) T_{n}^{-1}\right\| \leqq\left\|T_{n}^{-1}\right\|\left\|T_{n}-T_{0}\right\| .
$$

Since $\left\{\left\|T_{n}^{-1}\right\|\right\}$ is bounded, and $\left\|T_{n}-T_{0}\right\| \rightarrow 0$, we have that

$$
r_{0}\left(Y_{n+1}, Y_{0}\right) \rightarrow 0 \text {. }
$$

Therefore $Y_{n} \rightarrow Y_{0}$ relative to $d$. This completes the proof.

The completeness theorem for $\tilde{\theta}$ is shown in [4].

4.4 Theorem. Let $X$ be a Banach space. $\left(S_{X}, \widetilde{\theta}\right)$ is complete.

This result could also be obtained using the theorem on completeness of Hausdorff distance when the underlying space is complete (see page 198 of [10]), which gives the result that if $\left\{Y_{n}\right\}$ is a sequence Cauchy with respect to $\tilde{\theta}$, then $\tilde{\theta}\left(Y_{n}, Z\right) \rightarrow 0$, where the subspace $Z$ is

$$
\left\{x \in X \mid x=\lim _{n} y_{n}, \text { for some sequence }\left\{y_{n}\right\} \in \prod_{n=1}^{\infty} Y_{n}\right\}
$$

5. Partial converse to 3.2. The theorem of this section shows that under certain circumstances, $r_{0}$ can be made arbitrarily small by taking $\theta$ sufficiently small. We shall use a notion and a result occurring in [4].

Definition. The minimal angle $\phi^{(m)}(Y, Z)$ between two nonzero subspaces $Y, Z$ of a Banach space is defined by

$$
\sin \phi^{(m)}(Y, Z)=\inf _{\substack{y \in Y \\ \max \|\| y\|,\| z \mid\}=1}}\|y+z\|, \text { with } 0 \leqq \phi^{(m)}(Y, Z) \leqq \frac{\pi}{2} .
$$

The following result is valid: 
Let $Y$ and $Z$ be nonzero subspaces of a Banach space.

Then $Y \cap Z=\{0\}$ and $Y+Z$ is closed if and only if $\phi^{(m)}(Y, Z)>0$. Also of use will be the notion of angular distance [2].

Definition. Given two nonzero vectors $x, y$, we define the angular distance between them $\alpha[x, y]$ by

$$
\alpha[x, y]=\left\|\frac{x}{\|x\|}-\frac{y}{\|y\|}\right\| .
$$

The fact about angular distance which we shall need (Lemma 5.1 of [11]) is that for nonzero vectors $x, y$,

$$
\alpha[x, y] \max \{\|x\|,\|y\|\} \leqq 2\|x-y\| .
$$

Before proving 5.2 we point out that if one replaces (5.3) by the condition

$$
\tilde{\theta}\left(Y, Y^{\prime}\right)<\sin \phi^{(m)}(Y, Z),
$$

then a result in [4] gives the conclusion that $X$ is the direct sum of $Y^{\prime}$ and $Z$, from which the authors of [4] conclude the result 5.7. However, the important result (5.4) cannot be obtained from this development in [4], which supplies no quantitative information. Moreover, the following simple example shows that there are cases where the hypotheses of [4] do not hold and those of 5.2 do. Let $X$ be a Hilbert space and $x_{1}, x_{2}$ orthogonal unit vectors. Let $Y$ be the subspace spanned by $x_{1}$, let $Z=Y^{\perp}$, and for each complex number $\lambda$, let $Y_{\lambda}^{\prime}$ be the subspace spanned by $x_{1}+\lambda x_{2}$. Then $\sin \phi^{(m)}(Y, Z)=$ $\left\|P_{Y}\right\|=1$. It is straightforward to verify that for each $\lambda$,

$$
\theta\left(Y, Y_{\lambda}^{\prime}\right)=\frac{|\lambda|}{\sqrt{1+|\lambda|^{2}}} \text { and } \tilde{\theta}\left(Y, Y_{\lambda}^{\prime}\right)=\sqrt{2-\sqrt{\frac{2}{1+|\lambda|^{2}}}} .
$$

Thus the hypotheses of 5.2 are satisfied for all $\lambda$, while those of [4] are violated for sufficiently large $|\lambda|$.

5.2 Theorem. Let $X$ be a Banach space which is the direct sum of subspaces $Y$ and $Z$ with $Y \neq\{0\}, X$. Let $P_{Y}$ (resp., $\left.P_{Z}\right)$ be the projection on $Y$ along $Z$ (resp., on $Z$ along $Y$ ). If $Y^{\prime}$ is a subspace such that

$$
\theta\left(Y, Y^{\prime}\right)<\min \left\{\frac{1}{\left\|P_{Y}\right\|}, \sin \phi^{(m)}(Y, Z)\right\},
$$

then $X$ is the direct sum of $Y^{\prime}$ and $Z$. Furthermore, denoting by $P_{Y^{\prime}}$ the projection on $Y^{\prime}$ along $Z$, we have that $I-P_{Y}+P_{Y^{\prime}}$ is an invertible operator mapping $Y$ onto $Y^{\prime}$, and 
(5.4) $r_{0}\left(Y, Y^{\prime}\right) \leqq\left\|P_{Y}-P_{Y^{\prime}}\right\| \leqq\left\|P_{Z}\right\|\left\|P_{Y}\right\| \frac{\theta\left(Y, Y^{\prime}\right)}{1-\left\|P_{Y}\right\| \theta\left(Y, Y^{\prime}\right)}$

Proof. We first show that the sum of $Y^{\prime}$ and $Z$ is closed and direct. If $y^{\prime} \in \Sigma\left(Y^{\prime}\right)$ and $z \in \Sigma(Z)$, then for each $\delta>1$, there is a vector $y_{\delta}$ in $Y$ such that $\left\|y^{\prime}-y_{\delta}\right\| \leqq \delta \theta\left(Y, Y^{\prime}\right)$. Hence

$$
\alpha\left[y^{\prime}, z\right] \geqq\left\|z-y_{\delta}\right\|-\left\|y^{\prime}-y_{\delta}\right\| \geqq \sin \phi^{(m)}(Y, Z)-\delta \theta\left(Y, Y^{\prime}\right) .
$$

Letting $\delta \rightarrow 1^{+}$, we have

$$
\alpha\left[y^{\prime}, z\right] \geqq \sin \phi^{(m)}(Y, Z)-\theta\left(Y, Y^{\prime}\right)>0 .
$$

with the aid of (5.1), we infer that $\sin \phi^{(m)}\left(Y^{\prime}, Z\right)>0$.

To establish that $X$ is the direct sum of $Y^{\prime}$ and $Z$, we show that $Y \subseteq Y^{\prime}+Z$. Results obtained in the process will be used to establish (5.4). Let $\eta$ be any number such that $1<\eta<\left(\left\|P_{Y}\right\| \theta\left(Y, Y^{\prime}\right)\right)^{-1}$. For given $y_{0} \in Y$, we choose inductively two sequences $\left\{y_{j}\right\}_{j=0}^{\infty}$ in $Y$ and $\left\{y_{j}^{\prime}\right\}_{j=0}^{\infty}$ in $Y^{\prime}$ such that for each $j$,

$$
\left\|y_{j}-y_{j}^{\prime}\right\| \leqq \eta \theta\left(Y, Y^{\prime}\right)\left\|y_{j}\right\| \text { and } y_{j+1}=P_{Y}\left(y_{j}-y_{j}^{\prime}\right) \text {. }
$$

By induction,

$$
\left\|y_{j}\right\| \leqq\left(\left\|P_{Y}\right\| \eta \theta\left(Y, Y^{\prime}\right)\right)^{j}\left\|y_{0}\right\|, \text { for each } j \text {. }
$$

If we set $z_{j}=P_{z}\left(y_{j-1}-y_{j-1}^{\prime}\right)$ for $j \geqq 1$, then for each $j$,

$$
y_{j}=y_{j}^{\prime}+P_{z}\left(y_{j}-y_{j}^{\prime}\right)+P_{Y}\left(y_{j}-y_{j}^{\prime}\right)=y_{j}^{\prime}+z_{j+1}+y_{j+1} .
$$

Hence

$$
y_{0}=y_{0}^{\prime}+z_{1}+y_{1}=\cdots=\sum_{j=0}^{k}\left(y_{j}^{\prime}+z_{j+1}\right)+y_{k+1} .
$$

since $\eta \theta\left(Y, Y^{\prime}\right)\left\|P_{Y}\right\|<1$, it follows from (5.5) that $y_{j} \rightarrow 0$. Therefore

$$
y_{0}=\sum_{j=0}^{\infty}\left(y_{j}^{\prime}+z_{j+1}\right) \in Y^{\prime}+Z .
$$

in view of the continuity of $P_{Y^{\prime}}$ we have

$$
y_{0}=\sum_{j=0}^{\infty} y_{j}^{\prime}+\sum_{j=0}^{\infty} z_{j+1},
$$

from which it is clear that

$$
\left(P_{Y}-P_{Y^{\prime}}\right) y_{0}=y_{0}-\sum_{j=0}^{\infty} y_{j}^{\prime}=\sum_{j=0}^{\infty} z_{j+1} .
$$

From the definition of $z_{j+1}$, 


$$
\left\|z_{j+1}\right\| \leqq\left\|P_{Z}\right\|\left\|y_{j}-y_{j}^{\prime}\right\| \leqq\left\|P_{z}\right\| \eta \theta\left(Y, Y^{\prime}\right)\left\|y_{j}\right\| .
$$

Hence, using (5.5),

$$
\left\|z_{j+1}\right\| \leqq\left\|P_{Z}\right\|\left\|P_{Y}\right\|^{-1}\left(\left\|P_{Y}\right\| \eta \theta\left(Y, Y^{\prime}\right)\right)^{j+1}\left\|y_{0}\right\|
$$

Thus,

$$
\left\|\left(P_{Y}-P_{Y^{\prime}}\right) y_{0}\right\| \leqq\left\|P_{Z}\right\| \frac{\eta \theta\left(Y, Y^{\prime}\right)}{1-\left\|P_{Y}\right\| \eta \theta\left(Y, Y^{\prime}\right)}\left\|y_{0}\right\|
$$

Since $X$ is the direct sum of $Y$ and $Z$ and of $Y^{\prime}$ and $Z$, it is easy to see that $I-P_{Y}+P_{Y}$ is an invertible operator mapping $Y$ onto $Y^{\prime}$ and that for each $x \in X\left(P_{Y}-P_{Y^{\prime}}\right) P_{Y} x=\left(P_{Y}-P_{Y^{\prime}}\right) x$. If we let $\eta \rightarrow 1^{+}$in (5.6) and replace $y_{0}$ by $P_{Y} x$, we get

$$
\left\|\left(P_{Y}-P_{Y^{\prime}}\right) P_{Y} x\right\| \leqq\left\|P_{Z}\right\| \frac{\theta\left(Y, Y^{\prime}\right)}{1-\left\|P_{Y}\right\| \theta\left(Y, Y^{\prime}\right)}\left\|P_{Y} x\right\| \text {. }
$$

Since $\left(P_{Y}-P_{Y^{\prime}}\right) P_{Y} x=\left(P_{Y}-P_{Y^{\prime}}\right) x,(5.4)$ is immediate.

5.7 CoROLlary. $S_{X}^{\prime}=\left\{Y \in S_{X} \mid Y\right.$ is complemented $\}$ is open with respect to $\theta$. In fact, if $Y$ is complemented, and if $\theta\left(Y, Y^{\prime}\right)$ is sufficiently small, then $Y$ and $Y^{\prime}$ have a common complementary subspace.

5.8 COROLlaRY. If $Y$ has a complementary subspace, and if $\theta\left(Y_{n}, Y\right) \rightarrow 0$, then $r_{0}\left(Y_{n}, Y\right) \rightarrow 0$. Consequently, in view of (3.2) and (1.4), $r_{0}$ and $\theta$ define the same topology on $S_{X}^{\prime}$.

6. Further theorems on the structures $\left(S_{X}, d\right),\left(S_{X}, \tilde{\theta}\right)$ and comparison of $\theta$ with $d$.

6.1 THEOREM. The following statements are equivalent:

(1) $\left(S_{X}, d\right)$ is totally bounded.

(2) $\left(S_{X}, \tilde{\theta}\right)$ is totally bounded.

(3) $X$ is finite dimensional.

Proof. $\quad(1) \Rightarrow(2)$, by (3.2). If $X$ if infinite dimensional, then there is a sequence $\left\{Y_{n}\right\}$ of subspaces such that $Y_{n}$ has dimension $n$ and $Y_{n} \subset Y_{n+1}$. Consequently $\theta\left(Y_{n}, Y_{m}\right)=1$, for $n<m$, since $Y_{n}$ is a proper subspace of $Y_{m}$. Thus $\left\{Y_{n}\right\}$ has no Cauchy subsequence with respect to $\theta$. This shows that $(2) \Rightarrow(3)$. To complete the proof we show that $(3) \Rightarrow(2)$ and (1). We can, without loss of generality, assume that $X$ is a finite dimensional Hilbert space. Furthermore, since $d$ and $\tilde{\theta}$ determine the same uniformity on $S_{X}$, when $X$ is finite dimensional, it suffices to show that $(3) \Rightarrow(2)$. Let $\left\{Y_{n}\right\}$ be a sequence of subspaces, 
and let $\left\{P_{n}\right\}$ be the corresponding sequence of orthogonal projections. Since the closed unit sphere of $[X]$ is compact, $\left\{P_{n}\right\}$ has a Cauchy subsequence, and consequently the corresponding subsequence of $\left\{Y_{n}\right\}$ is Cauchy with respect to $\theta$. This completes the proof.

6.2 COROLlaRY. The following three statements are equivalent:

(1) $\left(S_{X}, d\right)$ is compact.

(2) $\left(S_{X}, \tilde{\theta}\right)$ is compact.

(3) $X$ is finite dimensional.

Proof. By 6.1 and the completeness theorems of $\S 4$.

The following observations are needed in what follows:

In a Banach algebra with identity $e$, the $\varepsilon$ sphere centered at $e$, for $\varepsilon \leqq 1$, is a convex set contained in the group of invertible elements, and hence this group is locally arcwise connected. It follows that the components of the group are arcwise connected.

We shall denote by $\mathscr{G}$ the group of invertible operators on the Banach space $X$, and by $\mathscr{G}_{1}$ the component of $I$ in $\mathscr{G}$.

6.3 TheOREM. $\left(S_{X}, d\right)$ is locally arcwise connected (and hence its components are arcwise connected).

Proof. Let $Y \in S_{X}$, and consider $\left\{Z \in S_{X} \mid r_{0}(Y, Z)<\varepsilon\right\}$, where $\varepsilon \leqq 1$. If $Z_{0}$ is in this set, there is an invertible operator $C$ mapping $Y$ onto $Z_{0}$ and such that $\|I-C\|<\varepsilon$. For each $\lambda \in[0,1]$, let $C_{\lambda}=$ $\lambda C+(1-\lambda) I$. Then each $C_{\lambda}$ is invertible, and $\left\|I-C_{\lambda}\right\|<\varepsilon$. Let $Y_{\lambda}=C_{\lambda} Y$, for each $\lambda$. Clearly $r_{0}\left(Y, Y_{\lambda}\right) \leqq\left\|I-C_{\lambda}\right\|<\varepsilon$. $Y_{\lambda^{\prime}}=$ $C_{\lambda^{\prime}} Y=C_{\lambda^{\prime}} C_{\lambda}^{-1} C_{\lambda} Y=C_{\lambda^{\prime}} C_{\lambda}^{-1} Y_{\lambda}$. Therefore $r_{0}\left(Y_{\lambda}, Y_{\lambda^{\prime}}\right) \leqq\left\|I-C_{\lambda^{\prime}} C_{\lambda}^{-1}\right\|$. Thus $\left\{Y_{\lambda}\right\}_{\lambda \in[0,1]}$ is an arc connecting $Y$ with $Z_{0}$ and lying in $\left\{Z \in S_{X} \mid r_{0}(Y, Z)<\varepsilon\right\}$. This completes the proof.

6.4 Theorem. Let $S_{X}^{\prime}$ be the set of complemented subspaces of $X$. Two subspaces $Y, Z$ in $S_{X}$ (resp., $S_{X}^{\prime}$ ) lie in the same component of $\left(S_{X}, d\right)\left(r e s p .,\left(S_{X}^{\prime}, d\right)\right)$ if and only if there is an operator $T$ in $\mathscr{G}_{1}$ such that $T Y=Z$.

Proof. We first prove the assertion for $S_{x}$. Define the relation " " on $S_{X}$ by:

$$
Y \sim Z \text { if and only if there is } T \in \mathscr{G}_{1} \text { such that } T Y=Z \text {. }
$$

It suffices to prove that it is an equivalence relation and that the equivalence classes modulo $\sim$ are components of $\left(S_{X}, d\right)$. Since $\mathscr{V}_{1}$ is a group, it is clear that $\sim$ is an equivalence relation. Let $\mathscr{C}$ be an 
equivalence class mod $\sim$. From the fact that the sphere of radius 1 about $I$ in $[X]$ is contained in $\mathscr{G}_{1}$, it can be seen that $\mathscr{M}$ is both open and closed. If $Y, Z \in \mathscr{M}$, and $T \in \mathscr{G}_{1}$, with $T Y=Z$, then in view of the arcwise connectedness of $\mathscr{G}_{1}$, there is an $\operatorname{arc}\left\{T_{\lambda}\right)_{\lambda \in[0,1]}$ lying in $\mathscr{G}_{1}$ such that $T_{0}=I, T_{1}=T$. It then follows that $\left\{T_{\lambda} Y\right\}_{\lambda \in[0,1]}$ is an arc lying in $\mathscr{M}$ connecting $Y$ with $Z$. Thus $\mathscr{M}$ is arcwise connected, and, being both open and closed, is not a proper subset of a connected set. This completes the proof for $S_{x}$. The details of the proof for $S_{X}^{\prime}$ are entirely analogous; one need only make the additional observation that an automorphic image of a complemented subspace is complemented.

6.5 CoRollary. If $\mathscr{G}$ is connected, then two subspaces $Y, Z$ in $S_{X}$ (resp., $\left.S_{X}^{\prime}\right)$ are in the same component of $\left(S_{X}, d\right)\left(\right.$ resp., $\left.\left(S_{X}^{\prime}, d\right)\right)$ if and only if there is an invertible operator $T$ mapping $Y$ onto $Z$.

6.6 Theorem. If $\mathscr{G}$ is connected, then two subspace $Y, Z$ in $S_{X}^{\prime}$ are in the same component of $\left(S_{X}^{\prime}, d\right)$ if and only if $Y$ is isomorphic to $Z$ and $X / Y$ is isomorphic to $X / Z$.

Proof. Any subspace complementary to $Y$ (resp., $Z$ ) is isomorphic to $X / Y$ (resp., $X / Z$ ) (in view of the theorem that a one-to-one bounded linear transformation of one Banach space onto another is an isomorphism). Hence by 6.5 the desired conclusion follows.

In a Hilbert space it follows from the polar decomposition theorem and the spectral theorem that any invertible operator is a product of two exponentials, and hence is connectable to $I$ by an arc. Hence if $X$ is a Hilbert space, $\mathscr{G}$ is connected. Since a finite dimensional normed linear space can be renormed with an equivalent norm which makes it a Hilbert space, $\mathscr{G}$ is also connected if $X$ is finite dimensional.

6.7 Corollary. If $X$ is finite dimensional, two subspaces $Y, Z$ are in the same component of $\left(S_{X}, d\right)$ or of $\left(S_{X}, \widetilde{\theta}\right)$ if and only if $\operatorname{dim} Y=\operatorname{dim} Z$.

6.8 Corollary. If $X$ is a Hilbert space, two subspaces $Y, Z$ are in the same component of $\left(S_{X}, d\right)$ or of $\left(S_{X}, \tilde{\theta}\right)$ if and only if orthogonal $\operatorname{dim} Y=$ orthogonal $\operatorname{dim} Z$ and orthogonal $\operatorname{dim} Y^{\perp}=$ orthogonal $\operatorname{dim} Z^{\perp}$.

Proof. By the theorem of [5] which states that if there is a oneto-one bounded linear transformation mapping the Hilbert space $H$ into the Hilbert space $K$, then orthogonal $\operatorname{dim} H \leqq$ orthogonal $\operatorname{dim} K$.

We remark that since $X$ and $\{0\}$ are isolated points of $\left(S_{X}, d\right)$ and $\left(S_{X}, \tilde{\theta}\right)$ neither of these metric spaces is connected if $X \neq\{0\}$. Less 
trivial is the fact that for a given positive integer $n,\left\{Z \in S_{X} \mid \operatorname{dim} Z=n\right\}$ is both open and closed with respect to $\theta$ (and hence with respect to d) by virtue of 2.1 .

The investigations of this paper pose the question, "Do $\theta$ and $d$ define the same uniformity on $S_{X}$, or, at least, the same topology?" We have seen in $\S 3$ that $\theta$ and $d$ do define the same uniformity on $S_{X}$, if $X$ is finite dimensional, or if $X$ is a Hilbert space. We also have 3.2 and the partial converse afforded by 5.2, as well as 5.7 and 5.8. In addition, we know that $S_{X}$ is complete with respect to either. A partial answer to the question, favoring the affirmative, would be that given a subspace $Y$, there is a positive $\delta$ such that $\theta\left(Y, Y^{\prime}\right)<\delta$ implies that $Y$ and $Y^{\prime}$ are isomorphic. In this connection, one might note the previously known theorems, 2.1-2.3, as well as 5.2. A still weaker partially affirmative answer would be that the set of subspaces which possess Schauder bases is open with respect to $\theta$, and it is to be noted that a proof that this is not generally valid would also prove (in view of 2.3) that there is a separable Banach space possessing no Schauder basis. The question as to whether every separable Banach space possesses a Schauder basis has been unsettled for many years.

7. The metric of Newburgh. In this section we depart from our previous conventions regarding the term "operator." An operator need not be bounded or everywhere defined, and can have its domain and range in different Banach spaces. In [12], Newburgh defines a metric $\delta$ on the set of nonzero subspaces of an arbitrary Banach space $X$. This metric is the Hausdorff metric associated with an appropriate metric $\rho_{X}$ defined on $X-\{0\}$. His purpose is to obtain a metric on the set of closed operators with domain in a Banach space $X_{1}$ and range in a Banach space $X_{2}$. This is accomplished by defining the distance between two such operators $T_{1}, T_{2}$ to be $\delta$ (graph $T_{1}$, graph $T_{2}$ ), where graph $T_{1}$, graph $T_{2}$ are considered as subspaces of the Banach space $X=X_{1} \times X_{2}$. We shall show in 7.1 that $\delta$ is equivalent to $\theta$. This gives a way of viewing $\delta$ and the results obtained with $\delta$ which is immediately and directly connected with the geometry of $X$, and eliminates the necessity of proceeding via the intermediate metric $\rho_{x}$. In view of Newburgh's results, it also provides applications of $\theta$ to the study of closed operators.

Before proving 7.1, we reproduce some of the machinery of [12].

Definition. Let $X$ be a Banach space, and let $X^{\prime}=X-\{0\}$. For any two vectors $a, b \in X^{\prime}$, define

$$
\begin{gathered}
\rho_{x}(a, b)=\inf \left\{\varepsilon \mid\|a-b\|<\left(e^{\varepsilon}-1\right)\|a\|\right. \\
\text { and } \left.\|a-b\|<\left(e^{\varepsilon}-1\right)\|b\|\right\} .
\end{gathered}
$$


$\rho_{x}$ is a metrix on $X^{\prime}$ defining the same topology as the norm.

Definition. Given two nonzero subspaces $Y, Z$ let $\delta(Y, Z)=$ $D(Y-\{0\}, Z-\{0\})$, where $D$ is the Hausdorff distance relative to the metric $\rho_{X}$ on $X^{\prime} . \quad \delta$ is always finite (in fact, $\leqq \log 3$ ), and hence is a metric on $S_{X}-\{\{0\}\}$.

7.1 THEOREM. Let $X$ be a Banach space, and $Y$ and $Z$ nonzero subspaces. Then

$$
\log (1+\theta(Y, Z)) \leqq \delta(Y, Z) \leqq \log (1+2 \theta(Y, Z)) .
$$

Proof. It is readily verified that for nonzero vectors $a, b$,

$$
\begin{aligned}
\rho_{x}(a, b) & =\inf \left\{\varepsilon \mid \log \left(1+\frac{\|a-b\|}{\|a\|}\right)<\varepsilon \text { and } \log \left(1+\frac{\|a-b\|}{\|b\|}\right)<\varepsilon\right\} \\
& =\max \left\{\log \left(1+\frac{\|a-b\|}{\|a\|}\right), \log \left(1+\frac{\|a-b\|}{\|b\|}\right)\right\} .
\end{aligned}
$$

In this proof, we shall continue to use " $\rho$ " to denote the norm distance of a point from a set, and we shall use " $\rho_{X}$ " in the usual senses. Let $y \in Y-\{0\}$. We shall show:

$$
\log \left(1+\frac{\rho(y, Z)}{\|y\|}\right) \leqq \rho_{X}(y, Z-\{0\}) \leqq \log \left(1+2 \frac{\rho(y, Z)}{\|y\|}\right)
$$

If $\left\{z_{n}\right\}$ is a sequence of vectors in $Z-\{0\}$ such that $\rho_{X}\left(y, z_{n}\right) \rightarrow$ $\rho_{x}(y, Z-\{0\})$, then for each $n$,

$$
\log \left(1+\frac{\left\|y-z_{n}\right\|}{\|y\|}\right) \leqq \rho_{x}\left(y, z_{n}\right)
$$

Hence

$$
\log \left(1+\frac{\rho(y, Z)}{\|y\|}\right) \leqq \rho_{x}\left(y, z_{n}\right), \quad \text { for each } n
$$

Letting $n \rightarrow \infty$, we obtain the first half of (7.3). By (5.1), for arbitrary $z \in Z-\{0\}$;

$$
\begin{gathered}
2 \frac{\|y-z\|}{\|y\|} \geqq \alpha[y, z]=\frac{\left\|y-\frac{\|y\|}{\|z\|} z\right\|}{\|y\|}, \\
\log \left(1+2 \frac{\|y-z\|}{\|y\|}\right) \geqq \log \left(1+\frac{\left.\left\|y-\frac{\|y\|}{\|z\|} z\right\|\right)}{\|y\|}\right)=\rho_{x}\left(y, \frac{\|y\|}{\|z\|} z\right) .
\end{gathered}
$$


The second half of (7.3) is immediate.

From (7.3) we conclude:

$$
\begin{aligned}
\log \left(1+\sup _{y \in \Sigma(X)} \rho(y, Z)\right) & \leqq \sup _{y \in Y-(0)} \rho_{X}(y, Z-\{0\}) \\
& \leqq \log \left(1+2\left(\sup _{y \in \Sigma(X)} \rho(y, Z)\right)\right) .
\end{aligned}
$$

Upon reversing the roles of $Y$ and $Z$, (7.2) follows.

It is interesting to note that:

7.4 Theorem. $\left(X^{\prime}, \rho_{X}\right)$ is complete, and hence the norm and $\rho_{X}$ define different metrizable uniformities on $X^{\prime}$ which induce the same uniform topology.

Proof. Suppose $\left\{x_{n}\right\}$ is Cauchy with respect to $\rho_{x}$. Then

$$
\frac{\left\|x_{n}-x_{m}\right\|}{\left\|x_{m}\right\|} \rightarrow 0, \text { as } n, m \rightarrow \infty .
$$

In particular, there is a positive integer $N$ such that $m, n \geqq N$ implies

$$
\left\|\frac{x_{n}}{\left\|x_{m}\right\|}-\frac{x_{m}}{\left\|x_{m}\right\|}\right\|<1 \text {. }
$$

So,

$$
\left|\frac{\left\|x_{n}\right\|}{\left\|x_{m}\right\|}-1\right|<1, \quad \text { for } m, n \geqq N \text {. }
$$

If either $\inf _{j}\left\|x_{j}\right\|=0$ or $\sup _{j}\left\|x_{j}\right\|=\infty$, then (7.6) leads to an absurdity. Thus

$$
\begin{gathered}
0<\inf _{j}\left\|x_{j}\right\| ; \\
\sup _{j}\left\|x_{j}\right\|<\infty .
\end{gathered}
$$

By (7.5) and (7.8), $\left\{x_{n}\right\}$ is Cauchy with respect to the norm. By (7.7), the limit in norm, $x$, of $\left\{x_{n}\right\}$ is not zero. Since $\left\|x_{n}-x\right\| \rightarrow 0$, it follows that $\rho_{X}\left(x_{n}, x\right) \rightarrow 0$. This completes the proof.

Given two Banach spaces $X_{1}, X_{2}$, we make $X_{1} \times X_{2}$ a Banach space by defining the algebraic operations coordinatewise, and setting $\left\|\left(x_{1}, x_{2}\right)\right\|=\left\|x_{1}\right\|+\left\|x_{2}\right\|$. We denote by $\mathscr{T}_{1,2}$ the set of closed operators with domain in $X_{1}$ and range in $X_{2}$, and by [ $\left.X_{1}, X_{2}\right]$ the set of bounded operators mapping all of $X_{1}$ into $X_{2}$. For operators $T_{1}, T_{2} \in \mathscr{T}_{1,2}$ we define 
$\theta\left(T_{1}, T_{2}\right)=\theta\left(\right.$ graph $T_{1}$, graph $\left.T_{2}\right)$, where graph $T_{1}$, graph $T_{2}$ are considered as subspaces of $X_{1} \times X_{2}$.

$\theta$ thereby defines a uniformity on $\mathscr{T}_{1,2}$. For $T \in \mathscr{T}_{1,2}$ we denote by $R(T)$ its range, and, if $T$ is one-to-one, $T^{-1}$ will be its set-theoretic inverse defined on $R(T)$. In this paragraph we present some of the results of [12] regarding $\mathscr{T}_{1,2}$, reformulated in terms of $\theta$. The continuity of an operator will mean continuity on its domain, and will not signity that the operator is everywhere defined.

(1) $\left\{T \in \mathscr{T}_{1,2} \mid T\right.$ is continuous $\}$ is open with respect to $\theta$.

(2) $\left\{T \in \mathscr{T}_{1,2} \mid T\right.$ is one-to-one, and $T^{-1}$ is continuous on $\left.R(T)\right\}$ is open with respect to $\theta$.

(3) $\left[X_{1}, X_{2}\right]$ is an open subset of $\mathscr{T}_{1,2}$ with respect to $\theta$.

(4) The set $S=\left\{T \in \mathscr{T}_{1,2} \mid T\right.$ is one-to-one, and $\left.T^{-1} \in\left[X_{2}, X_{1}\right]\right\}$ is open with respect to $\theta$, and the map which assigns to each operator in $S$ its inverse is continuous from $S$ with the topology induced by $\theta$ to $\left[X_{2}, X_{1}\right]$ with the uniform operator topology.

(5) The topology induced on $\left[X_{1}, X_{2}\right]$ by $\theta$ coincides with the uniform operator topology.

In conclusion, we point out that for a Banach space $X \neq\{0\}$, the uniformity determined by the operator norm on $[X]$ is not the same as that determined by $\theta$, and that $\mathscr{T}$, the set of closed operators with domain and range in $X$, is not complete with respect to $\theta$. In fact, for each positive integer $n$, let $T_{n}$ be the operator $n I$. One verifies that $\theta\left(\{0\} \times X\right.$, graph $\left.T_{n}\right) \leqq 1 / n$, for each $n$. Since \{graph $\left.T_{n}\right\}$ is Cauchy with respect to $\theta$, so is $\left\{T_{n}\right\}$; however, $\left\{T_{n}\right\}$ is obviously not Cauchy with respect to the operator norm. Moreover, although $\left\{T_{n}\right\}$ is Cauchy with respect to $\theta$, the fact that graph $\left.T_{n}\right\}$ tends to a subspace which is not a graph, shows that $\left\{T_{n}\right\}$ does not converge to an operator in $\mathscr{T}$ with respect to $\theta$.

\section{BIBILIOGRAPHY}

1. N. I. Achieser and I. M. Glasmann, Theorie der linearen Operatoren im HilbertRaum, Akademie Verlag, Berlin, 1954.

2. J. A. Clarkson, Uniformly convex spaces, Trans. Amer. Math. Soc., 40 (1936), 396-414. 3. I. C. Gochberg and M. G. Krein, Fundamental aspects of defect numbers, root numbers, and indexes of linear operators, Uspekhi Mat. Nauk., 12, 2(74) (1957), 43-118 (in Russian).

4. I. C. Gochberg and A. S. Markus, Two theorems on the opening between subspaces of a Banach space, Uspekhi Mat. Nauk., 89 (1959), 135-140 (in Russian).

5. P. R. Halmos and G. Lumer, Square roots of operators II, Proc. Amer. Math. Soc., 5 (1954), 589-595.

6. F. Hausdorff, Mengenlehre, Dover, New York, 1944.

7. E. Hille and R. S. Phillips, Functional Analyșis and Semi-Groups, Amẹr, Math. Soc, Colloq. Publ, 31 (1957). 
8. M. G. Krein and M. A. Krasnoselski, Fundamental theorems concerning the extension of Hermitian operators and some of their applications to the theory of orthogonal polynomials and the moment problem, Uspekhi Mat. Nauk., 2, 3 (1947) (in Russian). 9. M. G. Krein, M. A. Krasnoselski, and D. P. Milman, Concerning the deficiency numbers of linear operators in Banach space and some geometric questions, Sbornik Trudov Inst. A. N. Ukr. S. S. R., 11 (1948) (in Russian).

10. C. Kuratowski, Topologie I. Monografje Matematyczne, Warsaw-Lwow, 1933.

11. J. L. Massera and J. J. Schäffer, Linear differential equations and functional analysis, I, Annals of Math., 67 (1958), 517-573.

12. J. D. Newburgh, A topology for closed operators, Annals of Math., 53 (1951), 250-255.

13. A. E. Taylor, Introduction to Functional Analysis, John Wiley, New York, 1958.

\section{UNIVERSity OF CALIFornia at Los ANGeles}




\section{PACIFIC JOURNAL OF MATHEMATICS}

\section{EDITORS}

\section{RalPh S. Phillips}

Stanford University

Stanford, California

M. G. Arsove

University of Washington

Seattle 5, Washington
J. Dugundji

University of Southern California Los Angeles 7, California

Lowell J. Paige

University of California

Los Angeles 24, California

\section{ASSOCIATE EDITORS}
E. F. BECKENBACH
D. DERRY
H. L. ROYDEN
E. G. STRAUS
T. M. CHERRY
M. OHTSUKA
E. SPANIER
F. WOLF

\section{SUPPORTING INSTITUTIONS}

\author{
UNIVERSITY OF BRITISH COLUMBIA \\ CALIFORNIA INSTITUTE OF TECHNOLOGY \\ UNIVERSITY OF CALIFORNIA \\ MONTANA STATE UNIVERSITY \\ UNIVERSITY OF NEVADA \\ NEW MEXICO STATE UNIVERSITY \\ OREGON STATE UNIVERSITY \\ UNIVERSITY OF OREGON \\ OSAKA UNIVERSITY \\ UNIVERSITY OF SOUTHERN CALIFORNIA
}

STANFORD UNIVERSITY

UNIVERSITY OF TOKYO

UNIVERSITY OF UTAH

WASHINGTON STATE UNIVERSITY

UNIVERSITY OF WASHINGTON

AMERICAN MATHEMATICAL SOCIETY

CALIFORNIA RESEARCH CORPORATION SPACE TECHNOLOGY LABORATORIES

NAVAL ORDNANCE TEST STATION

Printed in Japan by International Academic Printing Co., Ltd., Tokyo Japan 


\section{Pacific Journal of Mathematics}

\section{Vol. 13, No. 1 \\ March, 1963}

Frantz Woodrow Ashley, Jr., A cone of super-(L) functions............. 1

Earl Robert Berkson, Some metrics on the subspaces of a Banach space....

Felix Earl Browder and Walter Strauss, Scattering for non-linear wave

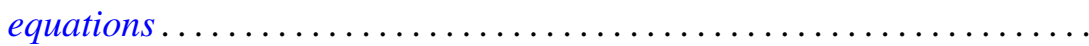

Edmond Darrell Cashwell and C. J. Everett, Formal power series ..........

Frank Sydney Cater, Continuous linear functionals on certain topological

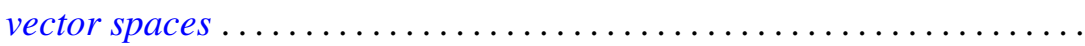

John Douglas Dixon, General group extensions ....................

Robert Pertsch Gilbert, On harmonic functions of four variables with

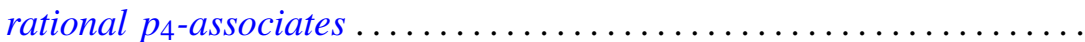

Irving Leonard Glicksberg, On convex hulls of translates ..............

Simon Hellerstein, On a class of meromorphic functions with deficient zeros

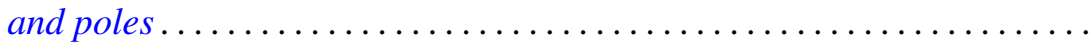

Donald William Kahn, Secondary cohomology operations which extend the

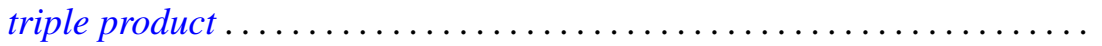

G. K. Leaf, A spectral theory for a class of linear operators .............

R. Sherman Lehman, Algebraic properties of the composition of solutions of partial differential equations ........................... 157

Joseph Lehner, On the generation of discontinuous groups ............. 169

S. P. Lloyd, On certain projections in spaces of continuous functions ...... 171 Fumi-Yuki Maeda, Generalized spectral operators on locally convex spaces ..................................

Donald Vern Meyer, $E^{3}$ modulo a 3-cell

William H. Mills, An application of linear programming to permutation groups.

Richard Scott Pierce, Centers of purity in abelian groups

Christian Pommerenke, On meromorphic starlike functions ...

Zalman Rubinstein, Analytic methods in the study of zeros of

polynomials...

B. N. Sahney, On the Nörlund summability of Fourier series

Tôru Saitô, Regular elements in an ordered semigroup . .

Lee Meyers Sonneborn, Level sets on spheres...........

Charles Andrew Swanson, Asymptotic estimates for limit point

problems .

Lucien Waelbroeck, On the analytic spectrum of Arens . .

Alvin (Murray) White, Singularities of a harmonic function of three

variables given by its series development .............

Kōichi Yamamoto, Decomposition fields of difference sets ...

Chung-Tao Yang, On the action of $\mathrm{SO}(3)$ on a cohomology manifold... 